\title{
Iterative Approach for the Clustering Problem *
}

\author{
Christophe Rapine and Denis Trystram \\ LMC-IMAG, BP 53, 38041 Grenoble Cedex 9, FRANCE
}

\begin{abstract}
This paper presents a new iterative approach of the clustering problem. We analyze the behaviour of the method applied on the Dominant Sequence Clustering (DSC) algorithm and prove that the parallel time of the series of clusterings built by the iterations is decreasing. We establish the optimality of the Iterative algorithm on outtrees and series-parallel graphs. Finally some experimental results are reported.
\end{abstract}

\section{Introduction}

The scheduling problem is known to be one of the most important challenge to take advantage of the whole computational power of parallel machines. It consists in determining an efficient execution of a program on a target system. One reasonable approach leads to modelize the parallel program as a set of computational tasks linked by precedence relations [1]. At compile time, the task partition of the program can be done in such a way to obtain a directed acyclic graph (dag) [7]. For the target machine, we adopt the model of RaywardSmith [6] which allows the overlapping of communication by computation and neglects the communication time between tasks located on the same processor. This model is quite realistic for topical message passing architectures. In addition we do not consider neither task duplication nor preemption. The purpose of this paper is to give an efficient solution for the scheduling problem allowing an unbounded number of processors. This problem called the clustering problem has been shown to be NP-hard $[5,8]$ in the presence of communication delays. The clustering algorithm we propose is based on an original iterative approach. The idea is to apply recursively the algorithm to build a series of clusterings whose parallel time decreases. We validate this approach using as basic brick the DSC algorithm of PYRROS [2] which we have extended to an iterative version, IDSC.

\subsection{Formalization of the Clustering Problem}

A valuated directed acyclic graph (dag) is a tuple $G=(X, E, v)$ where $X$ is a finite set of nodes. $E$, the set of arcs, is included into a partial order relation $\prec$ on $X$, and $v$, the valuation, is an application of $X \cup E \rightarrow I R^{+}$which associates to each node its computational cost and to each arc the amount of data to transfer. Node $x$ is a predecessor of $y$, or $y$ is a successor of $x$, if $(x, y) \in E$. The sets of successors and predecessors of a node $x$ are denoted respectively by $\operatorname{Succ}(x)$

* Part of the Apache project supported by CNRS, INRIA, INPG and UJF. 
and $\operatorname{Pred}(x)$. In case where $\operatorname{Pred}(x)=\emptyset$, respectively $\operatorname{Succ}(x)=\emptyset, x$ is called a root, respectively a leaf, of the graph. When all computation and communication costs are equal to 1, the graph is said $U E C T$. A clustering can be defined as a set $S=\left\{\left(X_{i},<_{i}\right)\right\}_{i \in \mathbb{N}}$ such that $\left(X_{i}\right)_{i \in N}$ is a partition of $X$. Each $X_{i}$, called cluster, represents the nodes allocated to the same processor $P_{i}$, and the $<_{i}$ is a total order relation on $X_{i}$ which determines the order of task execution. To verify the precedence constraints, $\left(\cup_{i \in \mathbb{N}}<_{i}\right) \cup \prec$ must be a partial order on $X$. In the case where each $<_{i}$ is included in $\prec$, the clustering is said linear, non-linear otherwise. We call clustered graph the graph where the communication delays between tasks of the same cluster have been zeroed. Adding pseudo-arcs of cost zero to represent the precedence relations $<_{i}$, we obtain the scheduled graph [3]. We call dominant sequence the critical path of the clustered graph. For linear clusterings, its length gives the completion time $P T(G, S)$ of the program. We denote finally by $S_{\emptyset}$ the clustering associating one cluster by task.

\subsection{Granularity}

The granularity of a graph tries to represent the relation between computation costs and communication delays. Basically if the granularity is greater than 1 (the graph is said coarse grain) the computation times will be preponderant in front of communications while for granularities lower than 1 the application will spend more time communicating than calculating. We adopt the notion of granularity introduced by Gerasoulis and Yang [3] which satisfies the property than any coarse grain dag admit a linear optimal clustering. The granularity $g$ of a dag $G=(X, E, v)$ is taken as the greatest real verifying for any node $x$ :

$$
\min _{y \in \operatorname{Pred}(x) \cup \operatorname{Succ}(x)}\{v(y)\} \geq g \cdot \max \left\{\max _{z \in \operatorname{Pred}(x)}\{v((z, x))\}, \max _{z \in \operatorname{Succ}(x)}\{v((x, z))\}\right\}
$$

\subsection{Iterative Approach}

Let denote by $\mathcal{S}$ the set of the clusterings of a $\operatorname{dag} G$. The clustering problem is interested in finding the subset $\mathcal{S}_{\text {opt }}$ of the optimal clusterings, i.e. the clusterings $S$ such that, for any clustering $R, P T(G, S) \leq P T(G, R)$. $\mathcal{S}_{\text {opt }}$ is the reciprocal image by $P T(G,$.$) of the minimal parallel time P T_{\text {opt }}(G)=\min \{P T(G, R) \mid R \in$ $\mathcal{S}$ \}. Roughly speaking, this definition leads to project the set $\mathcal{S}$ on $I R$. We want to give another characterization of $\mathcal{S}_{\text {opt }}$. The idea is to describe $\mathcal{S}_{\text {opt }}$ as the set of solutions of a general equation $F(S)=S$ with $F: \mathcal{S} \rightarrow \mathcal{S}$. Hence it is to find a function $F$ on $\mathcal{S}$ whose fixed points are exactly $\mathcal{S}_{\text {opt }}$. Moreover, in order to build an optimal solution, we would like that the iteration of function $F$ conducts to a fixed point. We follow this idea by iterating clustering algorithms. Indeed if we identify $G$ with the scheduled graph $\left(G, S_{\emptyset}\right)$, a clustering algorithm $a$ appears as a function of $\mathcal{S} \rightarrow \mathcal{S}$ defined only for $S_{\emptyset}$. It is natural to extend $a$ in such a way that it admits any clustering in entry. In addition we impose that $a$ must always improve the parallel time of the clustering $S$ it has in entry. This property ensures that the corresponding series of the parallel time $P T\left(G, a^{n} S\right)$ 
has a limit, we may hope near optimal. We choose for algorithm $a$ the DSC algorithm of PYRROS. We focus in the following on coarse grain graphs. For this reason we restrain ourself to the linear clusterings, known to be dominant on these graphs [3].

\section{DSC Algorithm}

The DSC algorithm [4] is based on a multi-step clustering refinement technic introduced by Sarkar [7]. Initially each task is assumed to be in a different cluster. A step consists in merging two clusters under the constraint that the parallel time does not increase. The choice of the arc to zero is achieved with a priority list heuristic based on the length of the longest path going through the nodes. To avoid an excessive time complexity, DSC visits the dag in a topological order: the graph is divided in two pieces, the examined graph (EG), already clustered, and the unexamined graph (UEG). At a step, when the partial solution $S$ has been build on EG, the length $P T(f, S)$ of the longest path going through node $f$ is the sum the greatest length $\operatorname{tlevel}(f, S)$ from a root to $f$, plus the greatest length blevel $(f, S)$ from $f$ to a leaf. Note that $\operatorname{tlevel}(f, S)$ is no more modified once $f$ has been examined, and $\operatorname{blevel}(f, S)=\operatorname{blevel}\left(f, S_{\emptyset}\right)$ until $f$ is examined. This allows a dynamic computation of the priority $\operatorname{Prio}(f)$, defined as $\operatorname{Prio}(f)=$ $p t l e v e l(f, S)+\operatorname{blevel}\left(f, S_{\emptyset}\right)$, where ptlevel $(f, S)=\max \{t l e v e l(y, S)+v(y)+$ $v(y, f)\}$ on $y$ the examined predecessors of $f$.

One step of DSC consists in choosing one free node, that is a node of UEG whose all predecessors have been examined, with the highest priority and zeroing one of its incoming arcs. Notice that for a free node we have ptelevel $(f, S)=\operatorname{tlevel}(f, S)$, and thus Prio $(f)$ is exactly $P T(f, S)$. Nevertheless the current dominant sequence may not go through a free node but a partially free node, i.e. an unexamined node with predecessors both in $E G$ and $U E G$. In this case the reduction of the tlevel of a free node $f$ may prevent a future reduction of the parallel time. Gerasoulis and Yang introduced the Dominant Sequence Reduction Warranty (DSRW): if $g$ is the partial free node with the highest priority and if the zeroing of the arc $(x, g)$ reduces ptlevel $(g)$ then no merging of $x$ with another free node $f$ is accepted if $\operatorname{Prio}(f)<\operatorname{Prio}(g)$.

\section{The Iterative Dominant Sequence Algorithm (IDSC)}

We extend $D S C$ in order that it accepts any linear scheduled graph $(G, L)$ as an entry, assuming $G$ coarse grain. The calcul of the priorities is done taking the blevel in $(G, L)$. To ensure the improvement of any solution, the constraint $D S R W$ has been enforced into a constraint $I D S R W$ : the zeroing of an arc $(x, f)$ is refused if there exists a successor $g$ of $x$, partially free, whose priority $\operatorname{Prio}(g)$ is equal to the priority of arc $(x, g)$ and strictly greater than the priority of arc $(x, f)$. The priority of an $\operatorname{arc}(x, y)$ is defined as Prio $(x, y)=\operatorname{tlevel}(x, S)+v(x)+$ $v(x, y)+\operatorname{blevel}(x, L)$, where $v$ is the valuation of graph $G, L$ the initial clustering and $S$ the current solution build by IDSC on EG. We choose to implement 
$\operatorname{IDSRW}(x, f)$ as a logic assertion, true if and only if the merge of $x$ and $f$ is acceptable: $\operatorname{IDSRW}(x, f)=\bigwedge(\operatorname{Prio}(x, g) \leq \operatorname{Prio}(x, f) \vee \operatorname{Prio}(x, g)<\operatorname{Prio}(g))$ on $g$ the partially free successors of $x$. The selection of node " $x$ " is done among the predecessors of $f$ which are not already merged, in such way that arc $(x, f)$ has the highest priority.

\section{Complexity}

The time complexity of DSC is in $O((m+n) \log m)$, with $n=|X|$ and $m=$ $|E|$. The verification of the constraint $I D S R W$ for an arc $(x, f)$ is done in time $O(|S u c c(x)|)$ which represents a total complexity of $O(n \cdot \max \{|\operatorname{Succ}(x)| \mid x \in$ $X\}) \subseteq O\left(n^{2}\right)$. As the complexity of DSC takes into account a multi-zeroing procedure which does not appear in IDSC as we focus on linear clusterings, the total time complexity of IDSC stays in $O\left(n^{2}\right)$.

Theorem 1. For any linear scheduled graph $(G, L)$, if idscL is a solution obtained by the application of IDSC algorithm, we have: $P T(G, i d s c L) \leq P T(G, L)$.

Proof. In the proof a clustering is identified to the set of its zeroed arcs. Let consider step $i$ when $i$ nodes have been examined. We denote by $E G_{i}$ and $U E G_{i}$ the current examined and unexamined graphs, by $S_{i}$ the current clustering built by IDSC, and by $L_{i}$ the clustering $L$ restricted to $U E G_{i}$. We introduce the clustering $S L_{i}$ formed by $S_{i} \cup L_{i}$ plus all the $\operatorname{arcs}(b, a) \in L$ such that $a \in U E G_{i}$, $b \in E G_{i}$ (the arc is said free) and no incoming arc of $a$ and no outgoing arc of $b$ belongs to $S_{i} \cup L_{i}$ in order to keep a linear clustering. If $(b, a)$ is a free arc, we have the property that $(b, a) \notin S L_{i} \Rightarrow P T\left((b, a), S L_{i}\right)=P \operatorname{Prio}_{i}(b, a)$, the priority at step $i$. We denote by $P T_{i}$ the parallel time $P T\left(G, S L_{i}\right)$, and by $P T_{i}() P T.\left(., S L_{i}\right)$. As $S L_{0}=L$ and $S L_{n}=i d s c L$, We propose to show that the $\left(P T_{i}\right)$ form a decreasing series. Let consider the transition of step $i$ to $i+1$ to establish that $P T_{i} \geq P T_{i+1}$ : it consists in selecting a free node $f$ and one of its predecessor, say $x$, and zeroing the arc $(x, f)$ under the constraint $I D S R W(x, f)$. Hence clustering $S L_{i+1}$ differs from $S L_{i}$ by the zeroing or the unzeroing of the arcs of the form $(x, g)$ and $(y, f)$. It is sufficient to show that the lengths of the longest paths of $\left(G, S L_{i+1}\right)$ going through such arcs are smaller than $P T_{i}$.

- Let $y \in \operatorname{Pred}(f) \backslash\{x\}$. As $f$ has been examined, we have $(y, f) \notin S L_{i+1}$. Let consider the case where $(y, f) \in S L_{i}$. Necessarily $(x, f) \notin S L_{i}$ and thus $\operatorname{Prio}_{i}(x, f)=P T_{i}(x, f) \leq P T_{i}$. Moreover we have $P T_{i+1}(y, f)=\operatorname{Prio}_{i}(y, f)$ and thus $P T_{i+1}(y, f) \leq \operatorname{Prio}_{i}(x, f) \leq P T_{i}$.

- Let $g \in S u c c(x), g$ free. If $(x, g) \notin S L_{i}$, then $P T_{i+1}(x, g)=P T_{i}(x, g) \leq$ $P T_{i}$. Otherwise $P T_{i+1}(x, g)=\operatorname{Prio}_{i}(x, g) \leq \operatorname{Prio}_{i}(x, f)=P T_{i}(x, f) \leq P T_{i}$.

- Let $g \in S u c c(x), g$ partially free. Let suppose that $\operatorname{IDSRW}(x, f)=$ TRUE. If $(x, g) \notin S L_{i}$, then $P T_{i+1}(x, g)=P T_{i}(x, g) \leq P T_{i}$. Otherwise, we have either $\operatorname{Prio}_{i}(x, g) \leq \operatorname{Prio}_{i}(x, f)$ which implies $P T_{i+1}(x, g) \leq P T_{i}(x, f) \leq$ $P T_{i}$, or $\operatorname{Prio}_{i}(x, g)<\operatorname{Prio}_{i}(g)$. In this case there exists an examined predecessor $x^{\prime} \neq x$ of $g$ such that $\operatorname{Prio}_{i}(g)=\operatorname{Prio}_{i}\left(x^{\prime}, g\right)$. Because of the linearity, $\left(x^{\prime}, g\right) \notin S L_{i}$, which implies that $\operatorname{Prio}_{i}\left(x^{\prime}, g\right)=P T_{i}\left(x^{\prime}, g\right)$, and thus 
$P T_{i+1}(x, g)<P T_{i}\left(x^{\prime}, g\right) \leq P T_{i}$. If $I D S R W(x, f)=$ FALSE, we know that there exists a partial free node $g^{\prime}$ successor of $x$ such that $\operatorname{Prio}_{i}(x, f)<\operatorname{Prio}_{i}\left(x, g^{\prime}\right)$ and $\operatorname{Prio}_{i}\left(x, g^{\prime}\right)=\operatorname{Prio}\left(g^{\prime}\right)$. We have only to check the longest path going through $(x, f)$ : if $(x, f) \notin S L_{i}$, then $P T_{i+1}(x, f)=P T_{i}(x, f) \leq P T_{i}$; otherwise necessarily $\left(x, g^{\prime}\right) \notin S L_{i}$, and thus $P T_{i}\left(x, g^{\prime}\right)=\operatorname{Prio}_{i}\left(x, g^{\prime}\right)=\operatorname{Prio}_{i}\left(g^{\prime}\right)$. It implies that $P T_{i+1}(x, f)=\operatorname{Prio}_{i}(x, f)<\operatorname{Prio}_{i}\left(x, g^{\prime}\right)=P T_{i}\left(x, g^{\prime}\right) \leq P T_{i}$.

In all cases the paths are bounded by $P T_{i}$, which implies that $P T_{i+1} \leq P T_{i}$.

\section{Optimality of IDSC}

\subsection{Coarse Grain Outtrees}

An outtree is a dag in which any node, except a special node called the root, has exactly one predecessor. On outtrees DSC may stay at $50 \%$ from the optimal time. Theorem 2 proves that IDSC reaches an optimal solution:

Theorem 2. For any coarse grain tree $T$ of height $h$ and any linear clustering $L$, we have $P T\left(T, i d s c^{h} L\right)=P T_{\text {opt }}(T)$.

Proof. First notice that the clustering of a subtree $T^{\prime}$ of $T$ by IDSC is independent from the rest of the tree. It implies that an optimal clustering on $T^{\prime}$ remains optimal after an iteration of IDSC. Hence if all the subtrees of $T$ are optimally clustered, one execution of IDSC will lead to the optimal for $T$ : the root of $T$ will be merged with its successor of longest path. Reasoning by induction, as the leaves are initially optimally clustered, in at most $h$ iterations IDSC finds an optimal solution.

\subsection{UECT SP-11 Graphs}

The series-parallel graphs are a generalization of the tree structures. The particular SP11 subclass, characterized by the fact that they have exactly one root and one leaf, modelizes well the remote procedure call paradigm. They can be recursively defined by the application of two operators, $\mathcal{S}$ and $\mathcal{P}$ : A single node $\circ$ is a SP11 graph, and if $\left(G_{i}\right)$ are SP11, $G_{1} \mathcal{S} G_{2}$ obtained by adding an arc between the leaf of $G_{1}$ and the root of $G_{2}$, and $G_{1} \mathcal{P} \ldots \mathcal{P} G_{k}$, obtained by adding a new node $r$ which becomes predecessor of the root of any $G_{i}$, and a new node $l$ which becomes successor of the leaf of any $G_{i}$, are SP11.

First we show that DSC may be at $33 \%$ from the optimal on SP11 graphs. Consider the recursive family of UECT SP11 graphs $\left(B_{n}\right)_{n}$ where $B_{0}$ is the graph ( $(\circ \circ) \mathcal{P}(\circ \mathcal{P} \circ)$ and $B_{n+1}$ is defined as $B_{n} \mathcal{P} L_{n}$, with $L_{n}$ a chain of $2 n+5$ nodes. A possible execution of DSC on $B_{n}$ will merge the root of $B_{i}$ with the root of $B_{i-1}$ for $i=1, \ldots, n$, leading to a parallel time of $3 n+7$. By induction we can show that the optimal parallel time is $2 n+7$. Hence we have $P T_{o p t}\left(B_{n}\right) \simeq$ $\left(1-\frac{1}{3}\right) P T_{d s c}\left(B_{n}\right)$. For IDSC, we have the convergence to the optimal solution on any coarse grain SP11 graph assuming that communication costs are all equal to the same constant $c$. Without lost of generality we take $c=1$ in the proof. 
Theorem 3. For any coarse grain UCT SP11 graph $G$ of height $h$ and any linear clustering $L$, we have $P T\left(G, i d s c^{\left\lceil\frac{h}{2}\right\rceil} L\right)=P T_{\text {opt }}(G)$.

Proof. The proof is done by induction on $h$ using the compositions $\mathcal{S}$ and $\mathcal{P}$. Let $G$ be a UECT SP11 graph of height $h$. If $h=0$, the result is obvious. Otherwise let suppose the result true for any graph of height $h^{\prime}<h$ :

- $G=G_{1} \mathcal{S} G_{2}$. We denote by $h_{1}$ and $h_{2}$ the heights of $G_{1}$ and $G_{2}$. As $h=h_{1}+h_{2}+1$, the theorem is true for $G_{1}$ and $G_{2}$. The behaviour of IDSC on $G_{1}$ (or $G_{2}$ ) is independent from the rest of $G$. Hence after $\max \left\{\left\lceil\frac{h_{1}}{2}\right\rceil,\left\lceil\frac{h_{2}}{2}\right\rceil, 1\right\}$ iterations, IDSC is optimal on $G$. This number of iterations is bounded by $\left\lceil\frac{h}{2}\right\rceil$.

- $G=G_{1} \mathcal{P} \ldots \mathcal{P} G_{k}$ with $k \geq 2$. We denote by $h_{i}$ the height of $G_{i}$. We have $h=\max \left\{h_{i} \mid 1 \leq i \leq k\right\}+2$. Hence, as the behaviour of IDSC on any subgraph $G_{i}$ is independent from the rest of the graph, in at most $\left\lceil\frac{h}{2}\right\rceil-1$ iterations each $G_{i}$ is optimally clustered. Let call $L^{\prime}$ the clustering $i d s c^{\left[\frac{h}{2}\right\rceil-1}(L)$. We have to prove that $P T\left(G, i d s c L^{\prime}\right)=P T_{\text {opt }}(G)$. As $i d s c L^{\prime}$ is still optimal on each $G_{i}, G_{i}$ can be seen as a single node $g_{i}$ of computation cost $P T_{o p t}\left(G_{i}\right)$. A simple case study according the parallel times $P T_{o p t}\left(G_{i}\right)$ permits to conclude to optimality.

\section{Experiments}

In this part we compare the behaviour of DSC and IDSC on grids. A d-dimensional grid, $\mathrm{dD}$ grid for short, of size $n$, is a graph constituted of $n^{d}$ nodes. A node is represented by a tuple $\left(i_{1}, \ldots, i_{d}\right), 1 \leq i_{j} \leq n$ and admits as successors the nodes $\left(i_{1}+1, i_{2}, \ldots, i_{d}\right), \ldots,\left(i_{1}, \ldots, i_{d-1}, i_{d}+1\right)$ if they exist. In the case of $3 \mathrm{D}$ grids, experiments show that IDSC converges quickly (cf figure 1) to the parallel time known to be equal to $5 n-4$ time units (the optimal improvement is asymptotically $16,7 \%$ as the initial time is equal to $6 n-5)$. To study the non UECT

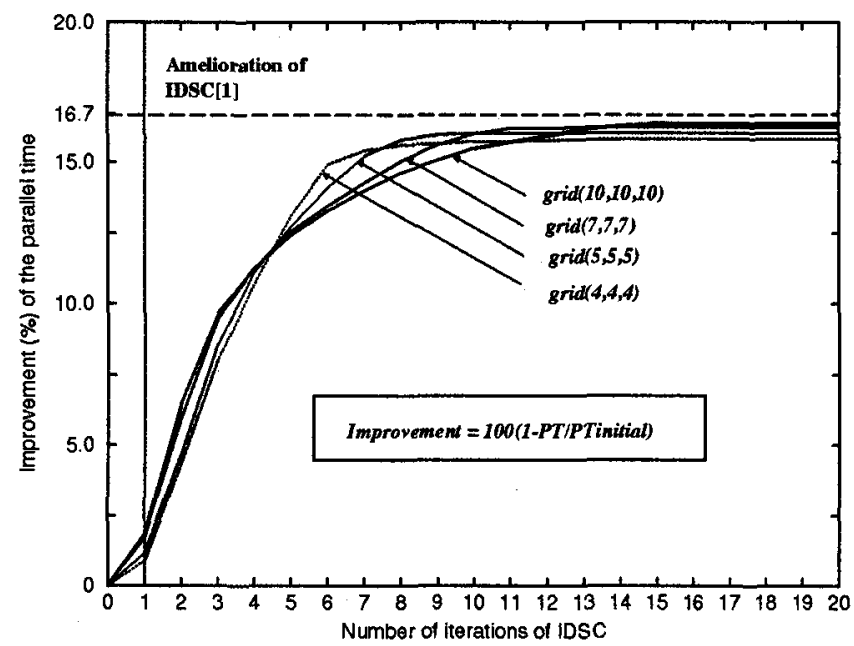

Fig. 1. Convergence of IDSC to the optimal clustering on $3 D$ UECT grids 
case, we generated 3D grids with communication costs all equal to a constant $c$ and computation costs randomly chosen in interval $[c, c+d]$, see figure 2 . The fraction $d / c$ represents the variation ratio of computations.

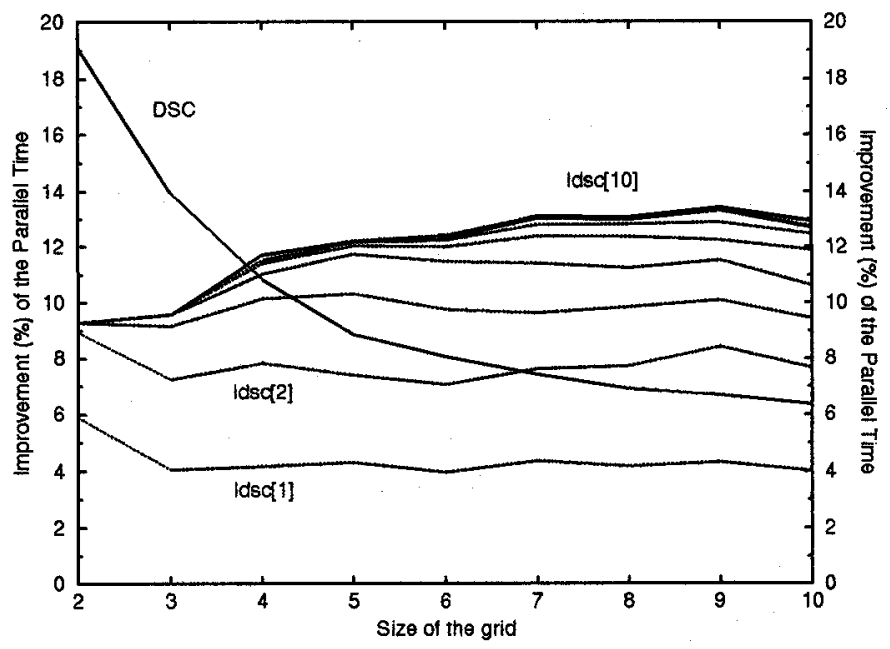

Fig. 2. behavior of DSC and IDSC on $3 \mathrm{D}$ grids with $50 \%$ computation cost variation.

One interesting question is the behaviour of the algorithm when the variation ratio $r$ becomes very small, say $r \ll 1$. This raises the problem of the stability: let consider a UECT $\operatorname{dag} G$, a clustering algorithm $a$ and the solution $S$ it computes. Let call $G_{\epsilon}$ the graph $G$ with its computation costs in intervals of the form $[1,1+\epsilon]$ with $\epsilon \ll 1$, and denote by $S_{\epsilon}$ the solution computed by $a$. These perturbations of computation costs may happen for instance if the valuation is obtained from monitoring: even if computation costs are theoretically equal, due to incertitude and system intrusions the measures will differ from one to another of some nanoseconds. One may hope, as $P T(G, S) \leq P T\left(G_{\epsilon}, S\right) \leq(1+\epsilon) P T(G, S)$, that $S_{\epsilon}$ will be closed to $S$, i.e. that $P T(G, S) \simeq P T\left(G_{\epsilon}, S_{\epsilon}\right)$. Experiments on perturbated 3D grids (figure 3 ) shows that it is not the case for DSC (nor IDSC). In order to estimate the optimal parallel time, we represent the clustering $L S$, optimal on the UECT grids, which merges all together the tasks of the same column. We give its performances in average, considering computation costs of $\left(1+\frac{r}{2}\right)$, and in the worst case, considering computation costs of $(1+r)$.

\section{Conclusion}

We have presented in this paper an iterative approach of the linear clustering problem. This idea has been illustrated on the well-know DSC algorithm. The theoretical analysis on some specific task graphs emphasizes the interest of 


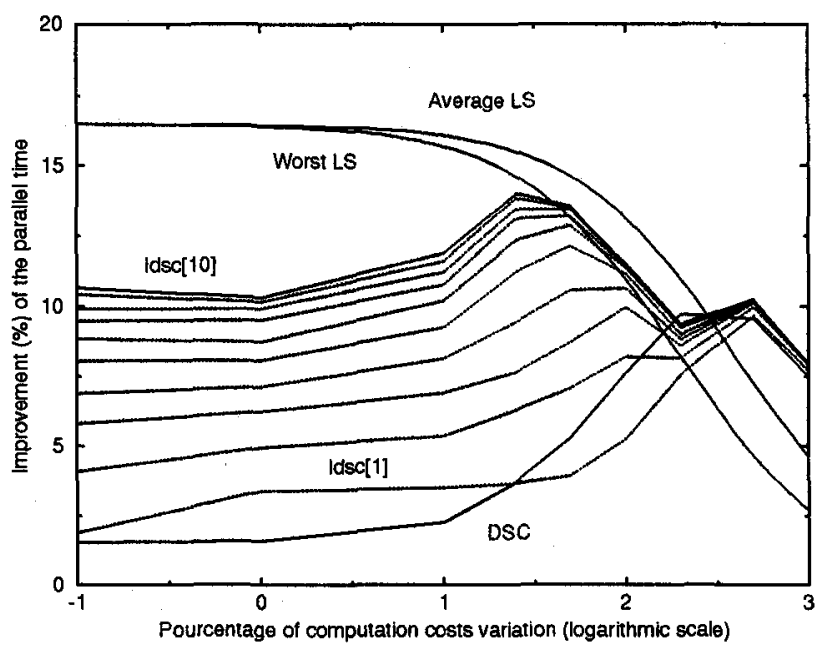

Fig. 3. Improvement of DSC and IDSC on 3D grids of size 15

such an approach. The experiments have shown the real potential of iterative methods, but obtaining the optimal solution remains an open question. We can think to mix this approach with a stochastic optimization algorithm like Monte Carlo methods. Another promisefull perspective would be to adapt this iterative approach to non-linear clusterings when the graph is not coarse grain.

\section{References}

1. E. Coffman and P. Denning. Operating System Theory. Prentice Hall, 1972.

2. A. Gerasoulis and T. Yang. PYRROS: static scheduling and code generation for message passing multiprocessors. In Proc. of the 6th ACM International Conf. on Supercomputing, pages 428-437. ACM, July 1992.

3. A. Gerasoulis and T. Yang. On the granularity and clustering of directed acyclic graphs. IEEE Trans. on Parallel and Distributed Systems, 4:186-701, 1993.

4. A. Gerasoulis and T. Yang. Dsc : Scheduling parallel tasks on an unbounded number of processors. IEEE Trans. on Parallel and Distributed Systems, 5:951-967, 1994.

5. C. Picouleau. Etude des Problèmes d'Optimisation dans les Systèmes Distribués. PhD thesis, Paris VI, Paris - France, 1993. in french.

6. V. Rayward-Smith. UET scheduling with unit interprocessor communication delays. Discrete Applied Mathematics, 18:55-71, 1987.

7. V. Sarkar. Partitioning and Scheduling Parallel Programs. Pitman, 1989.

8. B. Veltman. Multiprocessor scheduling with communication delays. $\mathrm{PhD}$ thesis, Univ. of Technology of Eindhoven, Depart. of Operations Research, May 1993. 